\title{
Monitoring the Behaviour of Credit Card Holders with Graphical Chain Models
}

\author{
Elena Stanghellini*
}

\section{INTRODUCTION}

Consumer credit is any of the many forms of commerce under which an individual obtains goods or services on condition of a promise to pay for their value, along with a fee (the interest), at some specific future date or dates. Consumer credit can take a variety of forms: bank loans, credit cards, retailer's card, car finance schemes, etc. In industrialized countries, this form of credit has become an enormous business. It is estimated that in Italy in the first semester of 1999, the total amount of consumer credits has increased by about $27 \%$ over the corresponding period in the previous year. This growth is in line with a positive trend started in the second half of the 1990s.

The need to cope with a vast demand for credits forced the credit grantors to implement automatic techniques for deciding whether to grant an application or not. Different information, coming from application forms, records of past behaviour and

\footnotetext{
* The author is from the Dipartimento di Scienze Statistiche, Università di Perugia, Italy. She is grateful to Catia Ciavarella for useful insights on the data set, and to Giovanni de Luca, Renato Paniccià and Nanny Wermuth for useful comments on a first draft of the paper. The paper has also benefitted by the reviewing process for which the comments of an Associate Editor and an anonymous referee are gratefully acknowledged. (Paper received November 2001, revised and accepted October 2002)
}

Address for correspondence: Elena Stanghellini, Dipartimento di Scienze Statistiche, Università di Perugia, Via Pascoli, 1 - C.P. 1315, Succ. 106100 - Italy.

e-mail: elena.stanghellini@stat.unipg.it 
credit reference bureaux, are processed to produce a 'score', that is a measure of the creditworthiness of each potential client or risk. Many statistical models, such as neural networks and logistic regressions have been implemented (for a review see Hand and Henley, 1997). When logistic regressions are used, for instance, the score is constructed as a monotonic transformation of the estimated odds of each applicant to be a good risk.

Models for applicant scoring are mainly univariate, their object being to provide the best classification rule according to a unique response. A crucial role is played by the definition of a good risk and by the choice of the predicting variables. In recent years, under the pressure of competition, finance agencies have started to develop new products. The aim is not only to widen their portfolio, but also to keep active relationships with good clients already taken on file and to prevent bad clients from becoming a loss for the agency. As an example of the first situation, it is more and more frequent that clients with one open relationship already with the credit grantors (for instance, a credit card) are offered a second loan with different schemes of repayment. As an example of the second situation, finance agencies have started to sell their own insurance on the loan. A bad client may still be profitable, provided that the client buys insurance on the loan and the defaults occur sufficiently late. As a result, models for the behaviour of the clients at different stages of the process are necessary. They involve many related outcome variables, which altogether give a measure of whether the client revealed to be profitable or unprofitable in the whole process.

This paper aims to show the potential of graphical chain models (see Lauritzen, 1996; or Edwards, 2000, for an extensive account) in the context described. We use a graphical chain model for monitoring the behaviour of people holding a credit card issued by a major Italian finance agency. Graphical chain models are used when there is a partial acyclic ordering between subsets of variables, such that variables in the first subset are merely explanatory, variables in the second subset are response for the previous ones and explanatory for the following ones, and so on.

There is a substantial proportion of clients who become inactive with the credit card. Early evaluation of which segments of the portfolio are most likely to become inactive is important 
information for the credit grantors, as it may determine which population to target with advertising campaigns. Also, evaluation of the policy with the credit limit is of interest, as it may reduce the number of drop-outs from the scheme.

Other applications of graphical models to credit scoring are in Hand et al. (1997), Sewart and Whittaker (1998) and Stanghellini et al. (1999). An excellent introduction to credit scoring together with more references can be found in the book of Hand and Jacka (1998). The issue of behavioural scoring is also addressed in the review article by Thomas (2000).

In Section 2 we review some of the properties of graphical chain models while in Section 3 we describe the data set and present a first ordering between the variables. A standard practice in credit scoring is to categorize the continuous variables. Here we categorize the continuous variables following the procedure proposed by Wermuth and Cox (1998). This categorization procedure is described in Section 3(i). In Section 3(ii) we illustrate the fitting procedure. Finally, in Section 4 we summarize the principal findings of the exercise and in Section 5 we draw some conclusions.

\section{GRAPHICAL CHAIN MODELS}

Graphical chain models are multivariate systems to study associations and dependencies among variables. They are used when a so called block-recursive structure can be deduced from prior information. This is a partial acyclic ordering between the variables such that variables in the first block are merely explanatory, variables in the last block are merely outcome, and variables in the intermediate blocks are response for the preceding ones and explanatory for the following ones.

Each model is represented by a graph where a node is associated to each variable and blocks are visualised by boxes (see Figure 1). Some pairs of nodes are joined by edges, which can be undirected, showing an association, or directed, showing a dependence. All edges in the same box are undirected, and all edges between different boxes are directed, the direction being the one specified by the ordering. A path connecting two nodes 
is a sequence of more than one undirected or directed edge which allows to go from one node to the other. A path without arrows between two nodes shows that the two variables are indirectly associated. A path with arrows going all in the same direction shows that the first variable in the ordering has an indirect influence on the second. The acyclic ordering implies that a variable cannot indirectly influence itself.

In models with only undirected edges, conditional independence between a pair of variables is visualized in the graph by the absence of an edge between the corresponding nodes. They have the nice property that the minimum conditioning set for each independence to hold can be readily visualised from the graph. For two nodes $i$ and $j$ not joined by an edge this is the subset of the remaining nodes such that each node of this subset is at least in one path connecting the two variables. This parallelism is due to the formal equivalence between Markov properties for undirected graphs and the graph-theoretic concept of separation (see Lauritzen, 1996, Ch. 3).

In a graphical chain model, the minimum conditioning set for each conditional independence can be visualized from the graph using a modified version of the separation criterion for undirected graphs. A lack of an undirected edge between the variables in the first box means that the pair of variables are independent giving the minimum conditioning set as defined for undirected graphs. For variables in the same box other than the first one, the variables in the preceding boxes should be added to the conditioning set to make a conditional independence statement. Finally, a lack of a directed edge between one variable and another variable in a later box means that the pair are independent given all the variables in the boxes preceding the one including the second variable.

These criteria can be sharpened (see Wermuth et al., 1998). Here we point out that conditional independence between a pair of variables, one in a box and the second one in a later box, means irrelevance of the information contained in the first variable to predict the second variable given the information on the variables in the minimum conditioning set. This can have an interesting implication in behavioural scoring, where recording and processing information can be costly and time consuming. 
In the literature, graphs have been used to represent different, though related, models (see Cox and Wermuth, 1996). The relationships between graphical models and other well-known multivariate statistical models are summarized in Wermuth (1992) and Cox and Wermuth (1993). For the relationships between graphical models and structural equation models see also Koster (1996).

\section{THE DETERMINANTS OF THE BEHAVIOUR WITH THE CREDIT CARD}

We analyze the behaviour of clients holding a credit card issued by a major Italian finance agency. The data set is formed by the people who started their business in the first six months of 1997 (about 60,000). The credit is so called 'revolving': for each client the amount of a new credit is summed over the previous ones. The monthly repayment is a fixed proportion of the total credits, which should not be higher than a threshold (the credit limit), plus the interest. Each month the client is classified as 'inactive' if the account has had no outstanding balance for more than six months, 'dormant' if the account has had no outstanding balance for six months or less, or 'active' if the account has an outstanding balance.

There is a substantial proportion (about $37 \%$ ) of these clients who after one year (the last measure available is 98/12) become inactive. About $18 \%$ are 'dormant' at $97 / 12$ (the behaviour around Christmas is an important variable) and, of these, about $73 \%$ become inactive at 98/12. As these clients have been selected by the agency through a scoring procedure, they are believed to be potentially good risks. Early evaluation of which segments of the portfolio are most likely to become inactive is important information for the credit grantor. This involves prediction of intermediate variables, i.e. variables which are not known at the time the client is taken on file, that are of interest and expected to have an impact on the purely outcome variables.

For each client a set of explanatory variables describing demographic and financial details is recorded. In this study we included a subset of these variables ('age', 'number of children', 
'residential code' and 'monthly income') and put them in the first box. These variables are used in the scoring system. The clients who entered the study have their score higher than a threshold. The selection strategy is therefore by conditioning on a response variable and the overall effect is to strengthen the interrelations among the explanatory variables.

One choice is therefore not to model their marginal distribution. However, we want to be able to draw conclusions also on subsets of population classified only on the basis of some of their demographic and financial characteristics. Therefore, the marginal model for them is of interest. The conclusions we draw are reliable as long as the selection strategy remains unchanged.

The credit limit is a deterministic function of the score and gives a measure of how good the client is supposed to be. It is therefore a function of the variables in the first box. We include it in the study as we want to be able to condition on its values. However, as the relationship between the credit limit and the previous variables is not of interest, we regard it as exogenous. In the graph, this is visualised by putting the node 'credit limit' in a separate doubly lined box at the same level of the explanatory variables. The variables in the following two boxes are, in order, the state of the account at 97/12 and at 98/12.

\section{(i) Categorization of the Variables}

Although it is possible to build models for continuous and discrete variables, we decided to categorize the continuous variables. This has been done by first defining a thin categorization, and then by merging levels of the explanatory variables when the corresponding local odd ratios in the $2 \times 3$ table with the last response variable (state of the account at 98/12) were not significantly different from one. The procedure we followed is described in Wermuth and Cox (1998). See also Agresti (1984).

As an example, we present the results of the analysis concerning the relevant categories of the number of children $(A)$. We first start with a thin categorization $(0,1,2,3$ or more) and consider the $4 \times 3$ table obtained from the cross classification of this variable $(A)$ with the state at 98/12 $(B)$. The entries are reported in Table 1 . We build the matrix of contrasts of log probabilities ordered with the column variable $(A)$ running 
Table 1

Observed Counts of the Cross-classification of Number of Children Against State at 98/12

\begin{tabular}{lrrrr}
\hline \multirow{2}{*}{ No. of Children } & Inactive & Dormant & Active & Sum \\
\hline 0 & 11,165 & 2,515 & 15,519 & 29,199 \\
1 & 5,107 & 1,255 & 7,832 & 14,194 \\
2 & 5,564 & 1,319 & 8,077 & 14,960 \\
$\geq 3$ & 1,694 & 354 & 2,940 & 4,988 \\
\hline Sum & 23,530 & 5,443 & 34,368 & 63,341 \\
\hline
\end{tabular}

fastest as a left Kronecker product of the matrix $C_{3}$ and $C_{4}$ so defined:

$$
C_{3}=\left(\begin{array}{rrr}
1 & 1 & 1 \\
-1 & 1 & 0 \\
0 & -1 & 1
\end{array}\right), C_{4}=\left(\begin{array}{rrrr}
1 & 1 & 1 & 1 \\
-1 & 1 & 0 & 0 \\
0 & -1 & 1 & 0 \\
0 & 0 & -1 & 1
\end{array}\right)
$$

The parameter corresponding, for instance, to the last column of the design matrix are log odds ratios of the $2 \times 2$ subtable for levels 2 and 3 of $A$ and levels 2 and 3 for $B$. We denote with $\gamma_{23,23}$ where the first two figures of the subscript refer to the corresponding levels of the $A$ variable.

In Table 2 the fitted studentized $\gamma$-parameters are reported. The ML estimates are obtained using the iteratively-reweighted

Table 2

Fitted Studentized $\gamma$-parameters

\begin{tabular}{lrr}
\hline & Saturated Model & Reduced Model \\
\hline$\gamma 12.12$ & 2.26 & 2.30 \\
$\gamma 23.12$ & -0.82 & 0.00 \\
$\gamma 34.12$ & -1.91 & -2.30 \\
$\gamma 12.23$ & 0.30 & 0.05 \\
$\gamma 23.23$ & -0.44 & 0.00 \\
$\gamma 34.23$ & 4.79 & 4.91 \\
\hline
\end{tabular}


least-squares algorithm. They show that the distinction between having one child or two children is irrelevant for predicting the state at 98/12: therefore the corresponding levels can be merged.

In Table 3 the categorization of the variables included in the analysis is presented. Note that 'residential code' takes value on a nominal scale. All the variables used have a strong marginal association with the response variable.

\section{Table 3}

Categories of the Variables in the Model

\begin{tabular}{lllrr}
\hline Age & No. of Children & Residential Code & $\begin{array}{c}\text { Credit Limit } \\
\text { (Euro) }\end{array}$ & $\begin{array}{c}\text { Monthly Income } \\
\text { (Euro) }\end{array}$ \\
\hline $18-44$ & 0 & Owner & $0-1033$ & $0-258.2$ \\
$45-54$ & 1 or 2 & Mortgage & $1034-3100$ & $258.3-516.5$ \\
& & Payer or Renter & & $516.6-774.8$ \\
55 or more & 3 or more & Other & & $\geq 774.9$ \\
\hline
\end{tabular}

\section{(ii) The Estimating Procedure}

A graphical chain model for categorical variables is fitted as a sequence of log linear models. This feature makes graphical chain models particularly attractive in the context of behavioural scoring, where log linear modelling has been widely employed.

First the log linear model for the variables in the first box is fitted, then the log linear model for the conditional distribution of the variables in the second box given the ones in the first box, then the log linear model for the conditional distribution of the variables in the third given the ones in the first two boxes, and so on. If at each step the estimates of the log linear model are ML, then the resulting model is ML (see Lauritzen, 1996, Ch. 4).

At each step, the matrix of contrast for the log probabilities of the $\log$ linear model has been built as a Kronecker product of $C$ matrices of level comparisons as previously described. As residential code is a nominal scaled variable of three categories, we build the corresponding matrix as: 


$$
C_{3}=\left(\begin{array}{rrr}
1 & 1 & 1 \\
-1 & 1 & 0 \\
-1 & 0 & 1
\end{array}\right)
$$

The log odds ratios involving this variable are therefore defined with the first category as a reference.

The fitted model for the variables in the first box contains some simplifications. There is independence in the $2 \times 2$ subtable obtained by the cross classification of the first two levels of 'income' and the second and third level of 'children'. This independence holds for any combinations of the other variables. Moreover, there is no three factor interaction between the third and fourth level of 'income' and the same levels of 'children' at any combinations for the other variables. However, the assumption of independence does not hold. The deviance for the selected model is 17.78 with 15 degrees of freedom.

The fitted model for the conditional distribution of the state at $97 / 12$ against the preceding variables has the following simplified structure. The interaction between the sociodemographic variables and the first response variable does not vary with the credit limit. Moreover, the interaction between 'age', 'residential code' and the response variable does not vary when passing from one child or two children to three or more. This holds for each combination of the remaining variables. The deviance of this selected model is 104.35 with 112 degrees of freedom.

The fitted model for the conditional distribution of the state at 98/12 against all the other variables shows that there is weak evidence that 'age' has no direct impact on this variable when information about the other variables is given. Due to the sparsity of the table, we evaluate the exact $p$-value for the deviance under this hypothesis (see Lauritzen, 1996, Ch. 4), which is equal to 0.011 . Although this assumption may be questionable, we feel that the gain made by reducing the dimensions of the table outweighs any resulting mis-specification of the model. The implication of this assumption is that 'age' has only indirect effects on the state at 98/12.

The selected model shows that the maximum order of interaction in this conditional distribution is the three-factor interaction. 
Also, the three-factor interaction terms not including the state at $97 / 12$ are not significant, but no further simplification can be achieved. In particular, the joint effect on the response variable of 'children', 'residential code' and 'T97/12' is highly significant (deviance 301.26 with 264 degrees of freedom). The deviance of the selected model is 233.18 with 237 degrees of freedom.

\section{PRINCIPAL FINDINGS}

The graphical chain model is reported in Figure 1. Inspection of the fitted distribution may lead to important conclusions. Here we summarize some of them. The estimated probability of being active at 98/12 varies substantially with the credit limit: from 0.38 for people with low credit limit to 0.68 for the ones with high credit limit. Also, the estimated probability of being active at both dates varies, from 0.12 in the first class to 0.36 in the second.

Income is estimated to have a negative effect on the probability of being active at 98/12. For instance, for high credit limit, the probability of being active at 98/12 decreases from 0.77 to 0.58 when passing from the lowest level of income to

\section{Figure 1}

The Graphical Chain Model for the Behaviour of Credit Card Holders

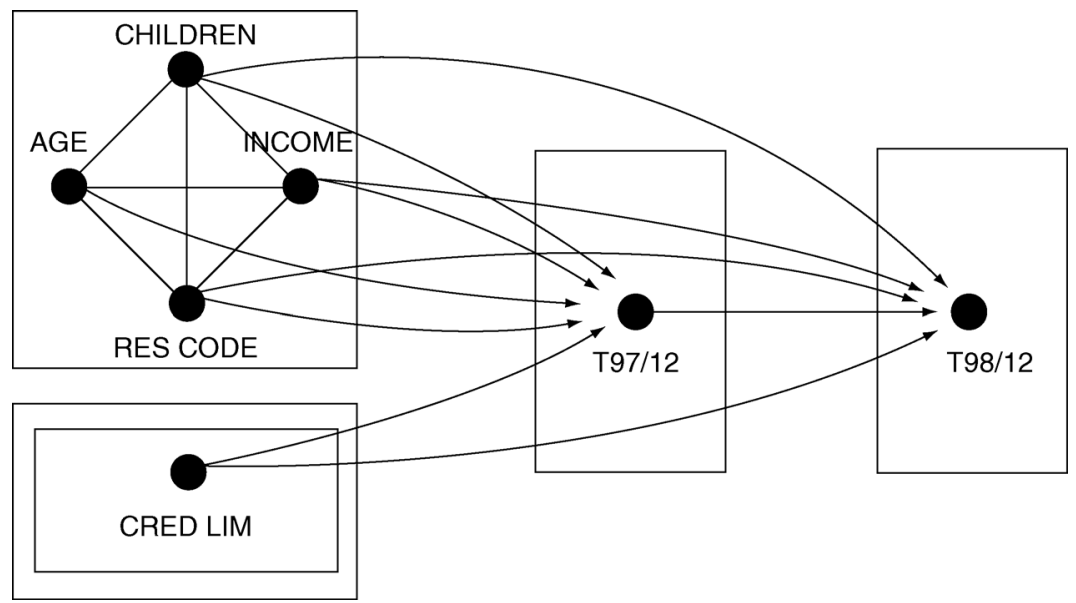


the highest. A parallel conclusion also holds for low credit limit, as the probability goes from 0.60 to 0.36 .

For people with low credit limit, the number of children has a strong impact on the probability of being active at 98/12 in the expected direction (from 0.36 to 0.44 when passing from no children to three or more). The effect of this variable on the state at 98/12, however, reduces when conditioning on home owners or people with high levels of income. The effect of having children is also reduced in people with high credit limit, going from 0.64 if no children to 0.67 if three children or more.

An optimal profile is shown by the people with high credit limit who are mortgage payer or renter. Their probability of being active at $97 / 12$ is 0.80 and 0.70 at $98 / 12$. This last probability raises to 0.76 if they have more than two children.

Early evaluation of which subgroups of the population are most likely to become inactive is important for the credit grantors, as it may determine which population to target with marketing campaigns. To this end, it is important to look at the conditional probability of the state at $98 / 12$ given the state at $97 /$ 12. As in the last model there is no interaction higher than the three-factor, for the analysis of this conditional probability it is sufficient to look at four-way marginal tables.

People with high credit limit, who are dormant at 97/12, have a higher probability of being active at $98 / 12$ than people with low credit limit (0.24 against 0.18$)$.

Looking at the two classes of credit limit separately, we found that for people with high credit limit who are home owners, this conditional probability is smaller (about 0.20 ) and does not vary when increasing the number of children. This effect is null also when conditioning on different classes of age.

This population, and in particular the one with children, may constitute an important population to target with advertising campaigns, as the high credit limit shows that they are considered potentially good risks. Also, the general trend in the population shows that children have a positive effect on the use of the credit card.

Parallel considerations concerning the joint effect of the explanatory variables on the conditional probability of being active at 98/12 if dormant at 97/12, can also be done for people 
with low credit limit. This is due to the vanishing of interaction terms higher than three-factor in the last model.

As these analyses show, there may be some gain in increasing the credit limit. As the credit limit is a measure of the creditworthiness of each client, the credit grantors may want to do it selectively, that is only on subgroups of the population having a good profile in terms of risks. The effect on this intervention may be evaluated from the model, by conditioning on the explanatory variables and allowing the credit limit to vary. The underlying assumption is that there is no association between the 'credit limit' and other unmeasured explanatory variables that are also associated with the response variables. This assumption must be supported from a priori considerations. As an example, for people aged between $45-54$, in the third level of income, who are home owners and have one child or two children (about $5 \%$ of the data set), the impact of this intervention in the probability of being active at 98/12 varies from 0.34 to 0.55 .

\section{CONCLUSIONS}

Models for behavioural scoring involve many related outcome variables. This paper explores the use of graphical chain models in monitoring the behaviour of credit card holders. We think that the modularity of these models, which allows to build them in blocks, is a point of strength for their use in the described situation, where distinction between intermediate and outcome variables can be crucial for an informative decision support.

\section{REFERENCES}

Agresti, A. (1984), Analysis of Ordinal Categorical Data (New York: John Wiley \& Sons).

Cox, D.R. and N. Wermuth (1993), 'Linear Dependencies Represented by Chain Graphs', Statistical Science, Vol. 8, No. 3, pp. 204-83.

— (1996), Multivariate Dependencies. Models, Analysis, and Interpretation (London: Chapman and Hall).

Edwards, D. (2000), Introduction to Graphical Modelling (2nd ed., New York: Springer-Verlag).

Hand, D.J. and W.E. Henley (1997), 'Statistical Classification Methods in Consumer Credit Scoring: A Review', Journal of the Royal Statistical Society, Series A, 160, pp. 523-41. 
Hand, D.J. and S.D. Jacka (eds.) (1999), Statistics in Finance (Arnold applications of statistics).

K.J. McConway and E. Stanghellini (1996), 'Graphical Models of Applicants for Credit', IMA Journal of Mathematics Applied in Business E Industry, Vol. 8, pp. 143-55.

Koster, J.T.A. (1996), 'Markov Properties of Nonrecursive Causal Models', Annals of Statistics, Vol. 24, pp. 2148-77.

Lauritzen, S.L. (1996), Graphical Models (Oxford: Oxford Science Publications).

Sewart, P. and J. Whittaker (1998), 'Graphical Models in Credit Scoring', IMA Journal of Mathematics Applied in Business E Industry, Vol. 9, pp. 241-66.

Stanghellini, E., D.J. Hand and K.J. McConway (1999), 'A Discrete Variable Chain Graph for Applicants for Credit', Applied Statistics, Vol. 48, Part 2, pp. 239-51.

Thomas, L.C. (2000), 'A Survey of Credit and Behavioural Scoring: Forecasting Financial Risk of Lending to Consumers', The International Journal of Forecasting, Vol. 16, pp. 149-72.

Wermuth, N. (1992), 'On Block-recursive Linear Regression Equations (with discussion)', Revista Brasileira de Probabilidade e Estatística, Vol. 6, pp. 1-56. - and D.R. Cox (1998), 'On the Application of Conditional Independence to Ordinal Data', International Statistical Review, Vol. 66, pp. 181-99. Derived from J. Pearl (1998), Explanations for Multivariate Structures subject to revision). 